\title{
Seoul Orthohantavirus in Wild Black Rats, Senegal, 2012-2013
}

Moussa M. Diagne, Idrissa Dieng, Laurent Granjon, Héloïse Lucaccioni, Abdourahmane Sow, Oumar Ndiaye, Martin Faye, Khalilou Bâ, Yamar Bâ, Mamoudou Diallo, Oumar Faye, Jean-Marc Duplantier, Mawlouth Diallo, Pascal Handschumacher, Ousmane Faye, Amadou A. Sall

Hantaviruses cause hemorrhagic fever in humans worldwide. However, few hantavirus surveillance campaigns occur in Africa. We detected Seoul orthohantavirus in black rats in Senegal, although we did not find serologic evidence of this disease in humans. These findings highlight the need for increased surveillance of hantaviruses in this region.

Tantaviruses (family Hantaviridae, genus Ortho1 hantavirus) are RNA viruses transmitted by aerosolized excreta from infected rodents and shrews. In humans, they cause hemorrhagic fever with renal syndrome (more often observed in Asia and Europe) and cardiopulmonary syndrome (more common in the Americas) (1). Only 1 case has been confirmed in Africa, in the Central African Republic in 1987 (2). However, studies from 2006 through 2013 have discovered new hantaviruses in autochthonous African rodents, moles, and bats $(3,4)$. In addition, serologic evidence in humans and rodents in Africa suggest local circulation (5). For example, a study in rural areas of Senegal found $11.5 \%$ of rodents and $16.6 \%$ of humans had antibodies against hantaviruses (3). More recently, serologic evidence of hantaviruses was reported in domestic and peridomestic rodents from some regions in Senegal (6).

Author affiliations: Institut Pasteur, Dakar, Senegal (M.M. Diagne,

I. Dieng, A. Sow, O. Ndiaye, M. Faye, Y. Bâ, Oum. Faye, Maw.

Diallo, Ous. Faye, A.A. Sall); Centre de Gestion des Populations, Institut de Recherche pour le Développement, Montpellier,

France (L. Granjon, J.-M. Duplantier); Université Paris Nanterre, Nanterre, France (H. Lucaccioni); Institut de recherche pour le développement Senegal, Dakar (K. Bâ, Mam. Diallo);

Sciences Economiques \& Sociales de la Santé \& Traitement de I'Information Médicale, Marseille, France (P. Handschumacher); Aix Marseille University, Marseille (P. Handschumacher); Institut National de la Santé et de la Recherche Médicale, Marseille (P. Handschumacher)

DOI: https://doi.org/10.3201/eid2610.201306
Southeastern Senegal has become a major trade area because of urbanization and substantial improvement of its road and rail networks in the late 1990s (7). Within a few years, these changes led to the rapid spread of a major invasive rodent species, the black rat (Rattus rattus [family Murinae]), which is a reservoir for Seoul orthohantavirus (SEOV) $(4,5,7)$. To assess the prevalence of hantaviruses in rodents, we screened for hantaviruses in $R$. rattus rats and commensal or peridomestic co-existing rodents in 20122013, approximately 15 years after the 1998 opening of a tarred road in eastern Senegal.

\section{The Study}

The national ethics committee for research of Senegal approved the study (authorization no. 0360-MSAS/ DPRS/DR, on October 24, 2011). During May 2012December 2013, we trapped small mammals as previously described (8) inside dwelling places and their surroundings (immediate and local) over periods of 1-6 consecutive days.

We caught 1,414 small mammals, including 403 black rats, from 10 different species (Appendix Table, https://wwwnc.cdc.gov/EID/article/26/10/201306-App1.pdf). We sampled whole blood, brain, and visceral organ tissues, which we then transferred to the Institut Pasteur (Dakar, Senegal). We triturated each solid sample in Leibovitz-15 medium (GIBCO-BRL, https://www.thermofisher.com) and centrifuged them to collect the suspension. To collect serum, we centrifuged whole blood samples. We extracted RNA from these different suspensions using the QIAamp RNA Viral Kit (QIAGEN, https://www.qiagen.com) according to the manufacturer's recommendations. To make cDNA, we used avian myeloblastosis virus reverse transcriptase (Promega, https://www.promega.com) followed by a nested conventional PCR with GoTaq Polymerase (Promega, https://www.promega. com) and a highly conserved hantavirus primers 
system selective for the partial large segment protein gene (9). We sequenced amplicons using GENEWIZ (https://www.genewiz.com), assembled them using EMBOSS Merger software (http://www.bioinformatics.nl/cgi-bin/emboss/merger), and analyzed them with BLAST (http://blast.ncbi.nlm.nih. gov/Blast.cgi). We performed sequence alignment with Mafft (10) and built a maximum-likelihood phylogenetic tree with iQ-TREE (11), using 1,000 replicates for bootstrapping.
Of the 1,414 mammals, 13 black rats tested positive for hantavirus RNA. We detected RNA in 14 samples: 9 brain homogenates, 4 multiorgan homogenates, and 1 serum sample. We confirmed the positive samples using PCR with highly conserved hantavirus small segment primers (12). Sequence analysis of partial large (deposited under GenBank accession nos. MT276868-81) and small (deposited under GenBank accession nos. MT276854-67) segments revealed 99.42\% identity withSEOV strain Rn-HD27 from China

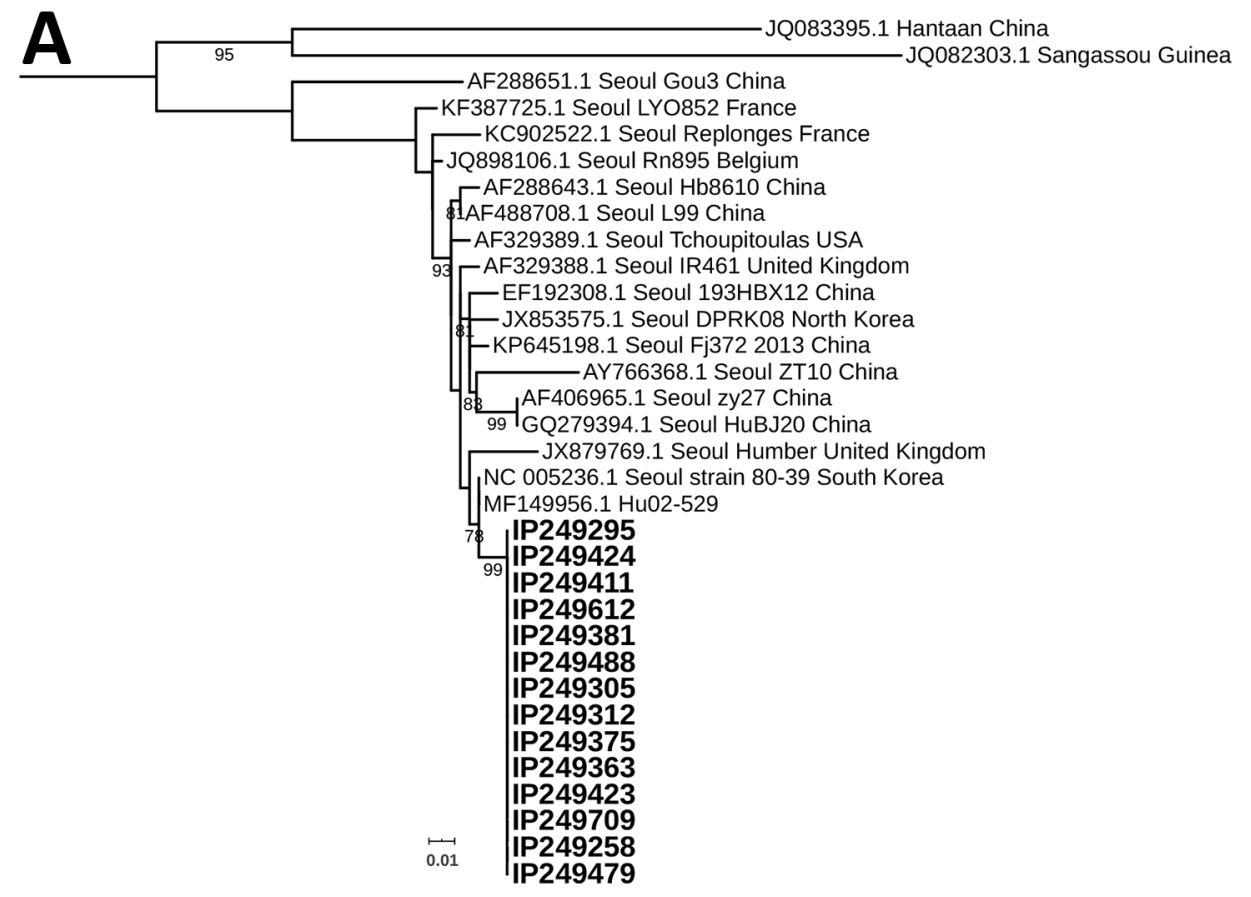

Figure 1. Phylogenetic analysis of Seoul orthohantavirus strains from black rats (Rattus rattus [family Murinae]; boldface) and reference sequences, Senegal, 2012-2013. Phylogenetic trees were generated by the maximum-likelihood method using the transition plus invariate sites plus gamma 4 model of the small segment (266 nt) (A) and the large segment (347 nt) (B). The numbers at each node are bootstrap probabilities ( $>90 \%$ ) as determined for 1,000 iterations. GenBank numbers are indicated for reference sequences. Scale bars indicate 0.01 substitutions per nucleotide $(A)$ and 0.1 substitutions per nucleotide (B).

NC 005222.1 Hantaan

B

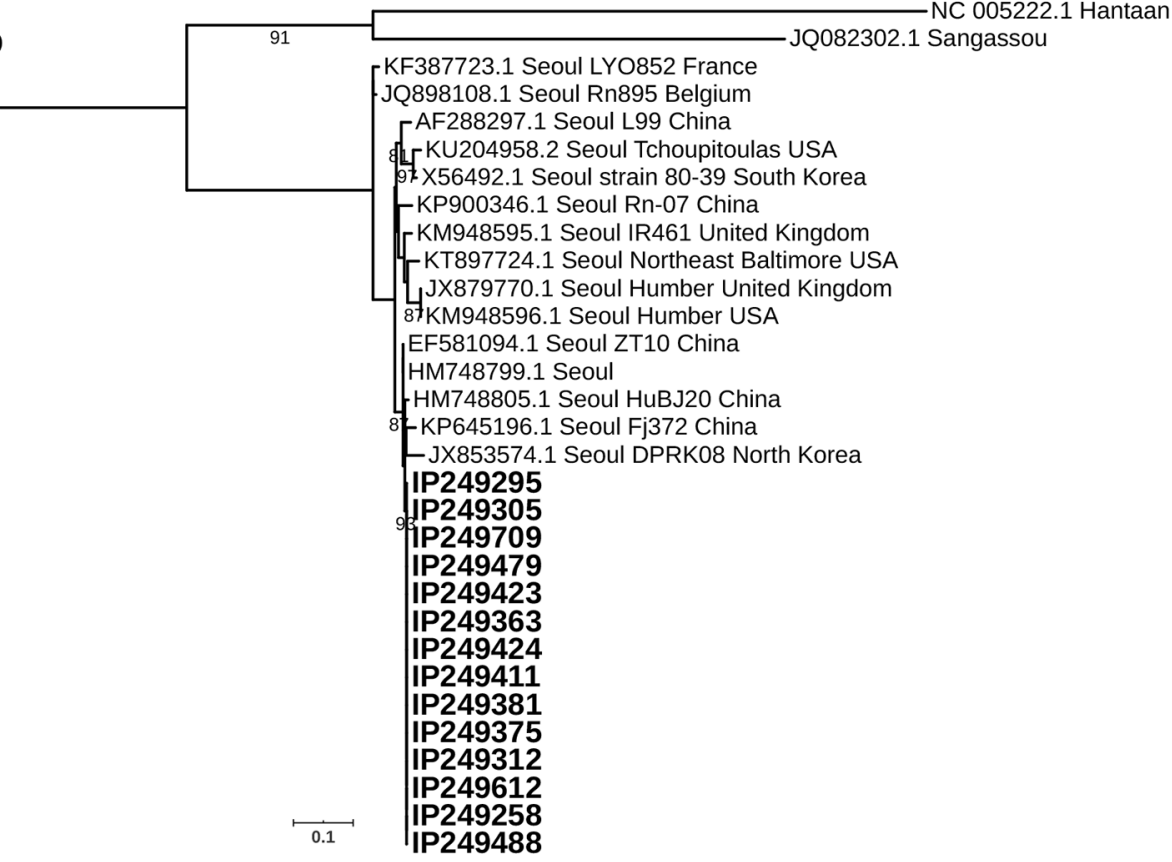


(GenBank accession no. HM748799) and 99.64\% identity with SEOV strain Hu02-529 from South Korea (GenBank accession no. MF149956) (Figure 1).

We detected SEOV RNA in 13 black rats caught in 3 villages: Goumbayel (7 rodents), Soutouta (4 rodents), and Dianke Makha (2 rodents). These villages were located $\approx 1$ hour's drive from the main road between Tambacounda and Kidira (Figure 2). Frequent movement of goods and humans between these 3 villages might explain the low genetic diversity among the new SEOV strains from black rats.
We did not observe signs of disease in the infected animals at the time of capture. Of the 4 villages that yielded the highest numbers of black rats in this study, 3 harbored rats infected with SEOV (Figure 2) (7). High densities of black rats might contribute to the occurrence of hantavirus in these villages, especially because host demography might affect hantavirus circulation (13).

Seasonal patterns might complicate these findings. We surveyed the villages harboring SEOVinfected rats in February 2013, which might be a
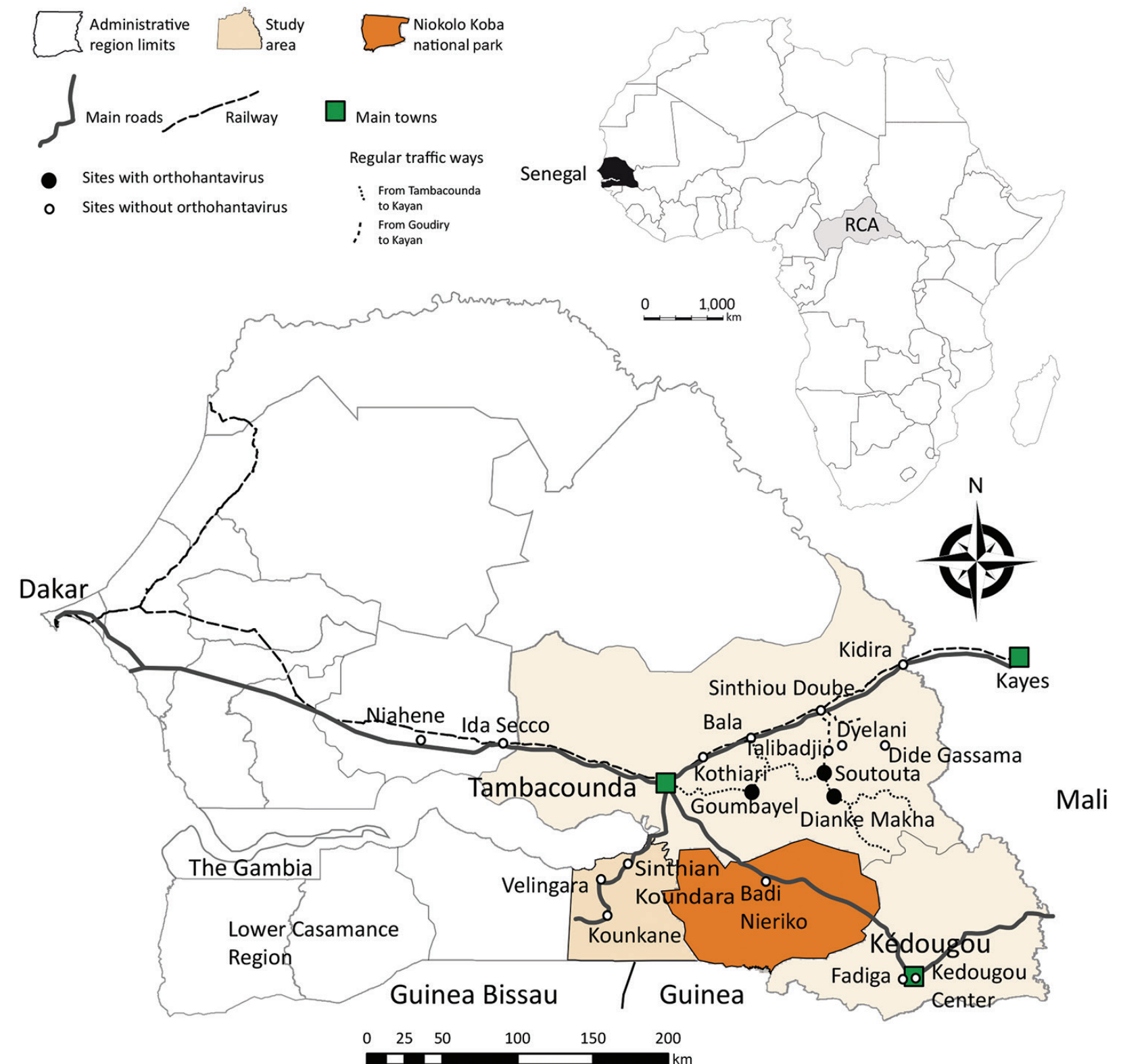

Figure 2. Locations of trapping sites (circles) used in study of rodentborne Seoul orthohantavirus in Senegal, 2012-2013. Black circles indicate trapping locations of Seoul orthohantavirus-infected black rats (Rattus rattus [family Murinae]). Inset shows location of Senegal in Africa. Map created using the package maptools installed in R studio version 1.2.1335 (https://rstudio.com/products/rstudio/) and shapefiles downloaded from the free domain of the Geographic Information System (http://www.diva-gis.org). 
Table. Human exposures to rodents in selected villages, Senegal, 2012-2013

\begin{tabular}{|c|c|c|c|c|c|}
\hline Village/town & $\begin{array}{c}\text { No. } \\
\text { participants }\end{array}$ & $\begin{array}{l}\text { No. }(\%) \text { participants in } \\
\text { contact with rodents }\end{array}$ & $\begin{array}{c}\text { No. distinct species } \\
\text { encountered }\end{array}$ & Black rats & Time period \\
\hline \multicolumn{6}{|l|}{ Tambacounda } \\
\hline Youpe Hamady & 87 & $70(80.5)$ & 4 & No & 2012 Oct $19-20$ \\
\hline Talibadji & 33 & $11(33.3)$ & 3 & Yes & 2012 Oct 21 \\
\hline Sinthiou Doube & 39 & 37 (94.9) & 4 & Yes & 2012 Oct 22 \\
\hline Ndiobene & 45 & $20(44.4)$ & 2 & No & 2012 Oct 22 \\
\hline Dianke Makha & 101 & $40(39.6)$ & 5 & Yes & 2012 Sep 10 \\
\hline Soutouta & 89 & $83(93.3)$ & 4 & Yes & 2012 Sep 11 \\
\hline \multicolumn{6}{|l|}{ Kedougou } \\
\hline Kedougou & 147 & $111(75.5)$ & 6 & Yes & 2013 Mar 9-10 \\
\hline Total & 541 & $372(68.8)$ & & & \\
\hline
\end{tabular}

favorable period for rodent reproduction, population increase, and thus hantavirus circulation (13). Despite the presence of juveniles, $R$. rattus populations had relatively high proportions of sexually active animals (75\% in Goumbayel, $48 \%$ in Soutouta, and $71 \%$ in Dianke Makha) (Appendix Figure). These data suggest that high level of interactions (male-female, adult-juvenile) occurred in these populations during that period, possibly promoting viral circulation. Conversely, we investigated nearby villages (Dieylani, Dide Gassama, Koussan, and Talibadji, in which we did not find evidence of hantavirusinfected black rats) in October 2012, at the end of the rainy season. Our investigations in May 2012 and November 2013 of the Kedougou area did not detect evidence of SEOV.

To assess potential human transmission, we performed parallel studies of human populations in some villages. Participants consented to an interview about rodent exposure and gave blood samples. During October 2012-March 2013, we recruited 541 participants with a mean age of 24 years (range 2-91 years) (Table). Of the 541 participants, $372(68.8 \%)$ reported close contact with rodents. The highest rates of rodent exposure were in Soutouta and Sinthiou Doube (Table). We performed an inhouse ELISA specific to IgG against SEOV on the human serum samples using reagents from the US Centers for Disease Control and Prevention (Atlanta, GA, USA). No IgG against SEOV was detected in the tested human samples, regardless of whether the participant's village had evidence of SEOV-infected black rats; this finding suggests a lack of human exposure. The role of species diversity in virus transmission is extremely complex (14). The relatively low SEOV prevalence in black rats (Appendix Table) might explain the negative results of the human serologic survey.

\section{Conclusions}

We found SEOV, a hantavirus pathogenic to humans, in black rats in southeastern Senegal. Phylogenic anal- yses grouped the newly detected SEOV with strains from Asia. Exchanges between Africa and Asia can potentially increase the opportunities for pathogens to expand their geographic range as previously described (15).

In-depth phylogenetic analysis of complete genomes would help elucidate the molecular evolution of this virus in Africa. This study highlights the need to improve hantavirus surveillance in Senegal and other countries in Africa for public health prevention strategies.

\section{Acknowledgments}

We thank Coly Bâ, Ambroise Dalecky, Christophe Amidi Diagne, Philippe Gauthier, Laëtitia Husse, Mamadou Kane, Youssou Niang, and Aliou Sow for their outstanding work during trapping sessions. We thank Oumar Ndiaye and Magueye Ndiaye for their technical laboratory support. We also thank Pierre Rollin for helping to obtain ELISA reagents.

This work was supported by grant nos. ANR-11-CEPL-0010 and ANR-11-JSV7-0006 (A.A.S., O.F., M.M.D., A.G., Y.B., M.D.) from the Agence Nationale de la Recherche.

\section{About the Author}

Dr. Diagne is a postdoctoral researcher at the Virology Department of Institut Pasteur de Dakar. His research interests include arboviruses and hemorrhagic fever viruses, such as hantaviruses in animal reservoirs and humans.

\section{References}

1. Bi Z, Formenty PB, Roth CE. Hantavirus infection: a review and global update. J Infect Dev Ctries. 2008;2:3-23. https://doi.org/10.3855/jidc.317

2. Coulaud X, Chouaib E, Georges AJ, Rollin P, Gonzalez JP. First human case of haemorrhagic fever with renal syndrome in the Central African Republic. Trans R Soc Trop Med Hyg. 1987;81:686. https:// doi.org/10.1016/0035-9203(87)90455-X

3. Witkowski PT, Klempa B, Ithete NL, Auste B, Mfune JKE, Hoveka J, et al. Hantaviruses in Africa. Virus Res. 2014; 187:34-42. https://doi.org/10.1016/j.virusres.2013.12.039 
4. Milholland MT, Castro-Arellano I, Suzán G, Garcia-Peña GE, Lee TE Jr, Rohde RE, et al. Global diversity and distribution of hantaviruses and their hosts. EcoHealth. 2018;15:163-208. https:/ / doi.org/10.1007/s10393-017-1305-2

5. Saluzzo JF, Digoutte JP, Adam F, Bauer SP, McCormick JB. Serological evidence for Hantaan-related virus infection in rodents and in Senegal. Trans R Soc Trop Med Hyg. 1985;79:874-5. https:// doi.org/10.1016/0035-9203(85)90145-2

6. Diagne CA, Charbonnel N, Henttonen H, Sironen T, Brouat C. Serological survey of zoonotic viruses in invasive and native commensal rodents in Senegal, West Africa. Vector Borne Zoonotic Dis. 2017;17:730-3. https://doi.org/10.1089/ vbz.2017.2135

7. Lucaccioni H, Granjon L, Dalecky A, Fossati O, Le Fur J, Duplantier J-M, et al. From human geography to biological invasions: the black rat distribution in the changing southeastern of Senegal. PLoS One. 2016;11:e0163547. https://doi.org/10.1371/journal.pone.0163547

8. Dalecky A, Ba K, Piry S, Lippens C, Diagne CA, Kane M, et al. Range expansion of the invasive house mouse $M u s$ musculus domesticus in Senegal, West Africa: a synthesis of trapping data over three decades, 1983-2014. Mammal Rev. 2015;45:176-90. https:/ / doi.org/10.1111/mam.12043

9. Klempa B, Fichet-Calvet E, Lecompte E, Auste B, Aniskin V, Meisel H, et al. Hantavirus in African wood mouse, Guinea. Emerg Infect Dis. 2006;12:838-40. https:/ / doi.org/10.3201/ eid1205.051487

10. Katoh K, Misawa K, Kuma K, Miyata T. MAFFT: a novel method for rapid multiple sequence alignment based on fast Fourier transform. Nucleic Acids Res. 2002;30:3059-66. https://doi.org/10.1093/nar/gkf436

11. Nguyen L-T, Schmidt HA, von Haeseler A, Minh BQ. IQ-TREE: a fast and effective stochastic algorithm for estimating maximum-likelihood phylogenies. Mol Biol Evol. 2015;32:268-74. https://doi.org/10.1093/molbev/msu300

12. Arthur RR, Lofts RS, Gomez J, Glass GE, Leduc JW, Childs JE. Grouping of hantaviruses by small (S) genome segment polymerase chain reaction and amplification of viral RNA from wild-caught rats. Am J Trop Med Hyg. 1992;47:210-4.

13. Tian H, Stenseth NC. The ecological dynamics of hantavirus diseases: from environmental variability to disease prevention largely based on data from China. PLoS Negl Trop Dis. 2019;13:e0006901. https:/ / doi.org/10.1371/ journal.pntd.0006901

14. Luis AD, Kuenzi AJ, Mills JN. Species diversity concurrently dilutes and amplifies transmission in a zoonotic hostpathogen system through competing mechanisms. Proc Natl Acad Sci U S A. 2018;115:7979-84. https://doi.org/10.1073/ pnas. 1807106115

15. Simon-Loriere E, Faye O, Prot M, Casademont I, Fall G, Fernandez-Garcia MD, et al. Autochthonous Japanese encephalitis with yellow fever coinfection in Africa. N Engl J Med. 2017;376:1483-5. https:/ / doi.org/10.1056/ NEJMc1701600

Address for correspondence: Moussa Moïse Diagne, Virology Department, Institut Pasteur de Dakar, 36 Avenue Pasteur, BP.220, Dakar, Senegal; email: MoussaMoise.DIAGNE@pasteur.sn

\section{EID Podcast Rabbit Fever in Organ Transplant Recipients}

In July 2017, three people developed tularemia, or "rabbit fever," after receiving organ transplants from the same donor. Donated organs are routinely screened for common viruses, but unusual diseases like tularemia can sometimes go undetected.

In this April 2019 EID podcast, Dr. Matthew Kuehnert, the medical director for the nation's largest tissue bank, MTF Biologics, explains how clinicians identified and diagnosed this rare disease.

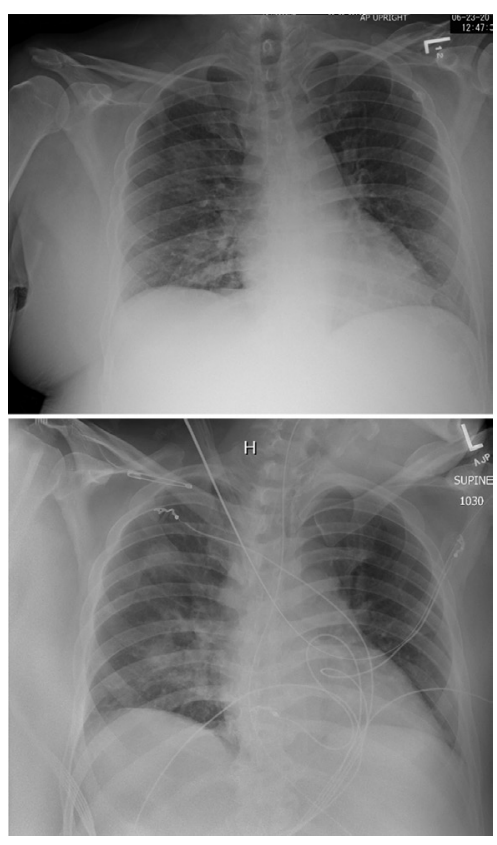

